\title{
REVIEW
}

\section{Autophagy and the nutritional signaling pathway}

\author{
Long HE ${ }^{1,2}$, Shabnam ESLAMFAM ${ }^{3}$, Xi MA $(\bowtie)^{1,2}$, Defa LI $(\bowtie)^{1}$ \\ 1 State Key Lab of Animal Nutrition, Ministry of Agriculture Feed Industry Center, China Agricultural University, Beijing 100193, China \\ 2 Center for Autophagy Research, Department of Internal Medicine and Biochemistry, Department of Biochemistry, \\ University of Texas Southwestern Medical Center, Dallas, TX 75390-9113, USA \\ 3 College of Dental Medicine, Midwestern University, Downers Grove, IL 60515, USA
}

\begin{abstract}
During their growth and development, animals adapt to tremendous changes in order to survive. These include responses to both environmental and physiological changes and autophagy is one of most important adaptive and regulatory mechanisms. Autophagy is defined as an autolytic process to clear damaged cellular organelles and recycle the nutrients via lysosomic degradation. The process of autophagy responds to special conditions such as nutrient withdrawal. Once autophagy is induced, phagophores form and then elongate and curve to form autophagosomes. Autophagosomes then engulf cargo, fuse with endosomes, and finally fuse with lysosomes for maturation. During the initiation process, the ATG1/ ULK1 (unc-51-like kinase 1) and VPS34 (which encodes a class III phosphatidylinositol (PtdIns) 3-kinase) complexes are critical in recruitment and assembly of other complexes required for autophagy. The process of autophagy is regulated by autophagy related genes (ATGs). Amino acid and energy starvation mediate autophagy by activating mTORC1 (mammalian target of rapamycin) and AMPactivated protein kinase (AMPK). AMPK is the energy status sensor, the core nutrient signaling component and the metabolic kinase of cells. This review mainly focuses on the mechanism of autophagy regulated by nutrient signaling especially for the two important complexes, ULK1 and VPS34.
\end{abstract}

Keywords Autophagy, ULK1 complex, VPS34 complex, AMPK, mTOR, nutrient signaling

\section{Introduction}

Autophagy is an autolytic process that degrades cellular

Received April 21, 2016; accepted June 16, 2016

Correspondences: maxi@cau.edu.cn,defali@cau.edu.cn components through lysosomes. In the 1960s, autophagosomes were first identified in mammalian cells by electron microscopy studies, but almost 30 years later, scientists uncovered the regulatory pathway and molecular mechanism. Following the discovery of the first autophagy genes (ATGs) in yeast, more than 30 ATG (autophagyrelated) genes required for autophagy and its related pathways have been identified ${ }^{[1]}$. Autophagic degradation can be classified as macro-autophagy, micro-autophagy and chaperone-mediated autophagy, depending mostly on the means of cargo delivery to the lysosome ${ }^{[2]}$. Macroautophagy is usually referred to as autophagy. Phagophores (isolation membranes) engulf the cytoplasmic components then elongate and curve to form closed double-membrane structure known as autophagosomes. Finally, the autophagosome delivers its cargo to fuse with lysosomes for degradation ${ }^{[3]}$. The two important complexes that regulate autophagosome biogenesis are ULK1 complexes and the class III phosphatidylinositol 3-kinase (PI3K) complex. Autophagy can be induced by nutrient deprivation, protein aggregates, organelle damage and pathogen infection. During nutrient signaling, $\operatorname{mTOR}^{[4]}$ and $\mathrm{AMPK}^{[5]}$, which sense the amino acid or energy status master kinase, directly affect the autophagy activity through the two complexes above. Under certain conditions such as birth ${ }^{[6]}$ and weaning ${ }^{[7]}$, transient starvation and stress will induce autophagy. Mice lacking ATG5 or ATG7, which are the essential autophagy genes, will decrease plasma and tissue amino acid concentrations and will die within a day of birth ${ }^{[8,9]}$. During stress, autophagy maintains cellular amino acids and ATP level for protein synthesis and supplying substrates for the tricarboxylic acid (TCA) cycle ${ }^{[10]}$. More recently, autophagy has been shown to be involved in the recognition and degradation of intracellular pathogens and functions as an infection barrier $^{[11,12]}$. Bacterial targets of autophagy include the intestinal pathogens, e.g., Salmonella typhimurium ${ }^{[13-15]}$. Autophagy in intestinal epithelial cell seems to be essential

(C) The Author(s) 2016. Published by Higher Education Press. This is an open access article under the CC BY license (http://creativecommons.org/licenses/by/4.0) 
for mammalian intestinal defense against invasive bacteria $^{[16]}$. This review aims to summarize and dissect the autophagy mechanism and interplay of the nutrient signaling pathway and pathogen recognition and defense.

The pre-autophagosomal structure (PAS/phagophore) is a single punctate structure close to the yeast vacuole membrane, where almost all Autophagy related gene (ATG) proteins are colocalized. In response to amino acid starvation or Adenosine triphosphate (ATP) withdrawal, the mechanistic Target Of Rapamycin (mTOR) will be inhibited, the 5' AMP-activated protein kinase (AMPK) will be activated to regulate initiation of autophagy by affecting the activity of Unc-51 Like Autophagy Activating Kinase 1 (ULK1) and Phosphatidylinositol 3-kinase (VPS34) complexes. Upon phagophore assembly, many genes are assembled into core autophagy machinery. VPS34 complex phosphorylates PtdIns (phosphatidylinositol) to PtdIns3P [phosphatidylinositol-(3)-phosphate] which recruit many binding proteins, such as Autophagy related gene 18 (ATG18), to membranes; Autophagy related gene 9 (ATG9) is the transmembrane protein carrying membrane for phagophore expansion. LC3, a homolog of yeast Atg8, localizes to autophagosomal membranes after post-translational modifications and conjugates with phosphatidylethanolamine (PE) by a ubiquitin-like system. Autophagy related gene 12 (ATG12)-modified Autophagy related gene 5 (ATG5) forms a multimeric complex with Autophagy related gene 6 (ATG16) which decorates the expanding phagophore with LC3-PE. When the autophagic doublemembrane engulfs its cargo and closes, the autophagosome will be fused with the lysosome. Many proteins: VesicleAssociated Membrane Protein 7, 3, 8 (VAMP7,3,8), QaSNARE syntaxin 17 (STX17), Synaptosome associated protein 29(SNAP-29) or other proteins such as Vesicle transport through interaction with t-SNAREs $1 \mathrm{~B}(\mathrm{Vti} 1 \mathrm{~b})$, BECN1-associated autophagy-related key regulator, Barkor or ATG14, CoCrMo metal particles (COPs), Endosomal complex enquired for transport III (ESCRTIII), Homotypic fusion and protein sorting (HOPS), Lysosomal associated membrane protein 2 (LAMP-2), Ras-related protein 7 (Rab7), Tectonin beta-propeller repeat containing 1 (TECPR1), Autophagy related gene 5, 12 (ATG5, ATG12), RUN and cysteine rich domain containing beclin 1 interacting protein (Rubicon) and UV radiation resistance associated gene (UVRAG)-associated VPS34 complex are involved in this process. Finally, the autophagosome cargo is delivered to be digested in the lysosome.

\section{Mechanism of autophagosome formation and maturation}

In canonical macro-autophagy, hereafter referred to as autophagy, the membrane derived from the endoplasmic reticulum separates to form the phagophore, then elongates and curves to form a closed double-membrane structure, called the autophagosome. The autophagosome engulfs cargos to fuse with endosomes, and then finally fuses with lysosomes to mature (Fig. 1).

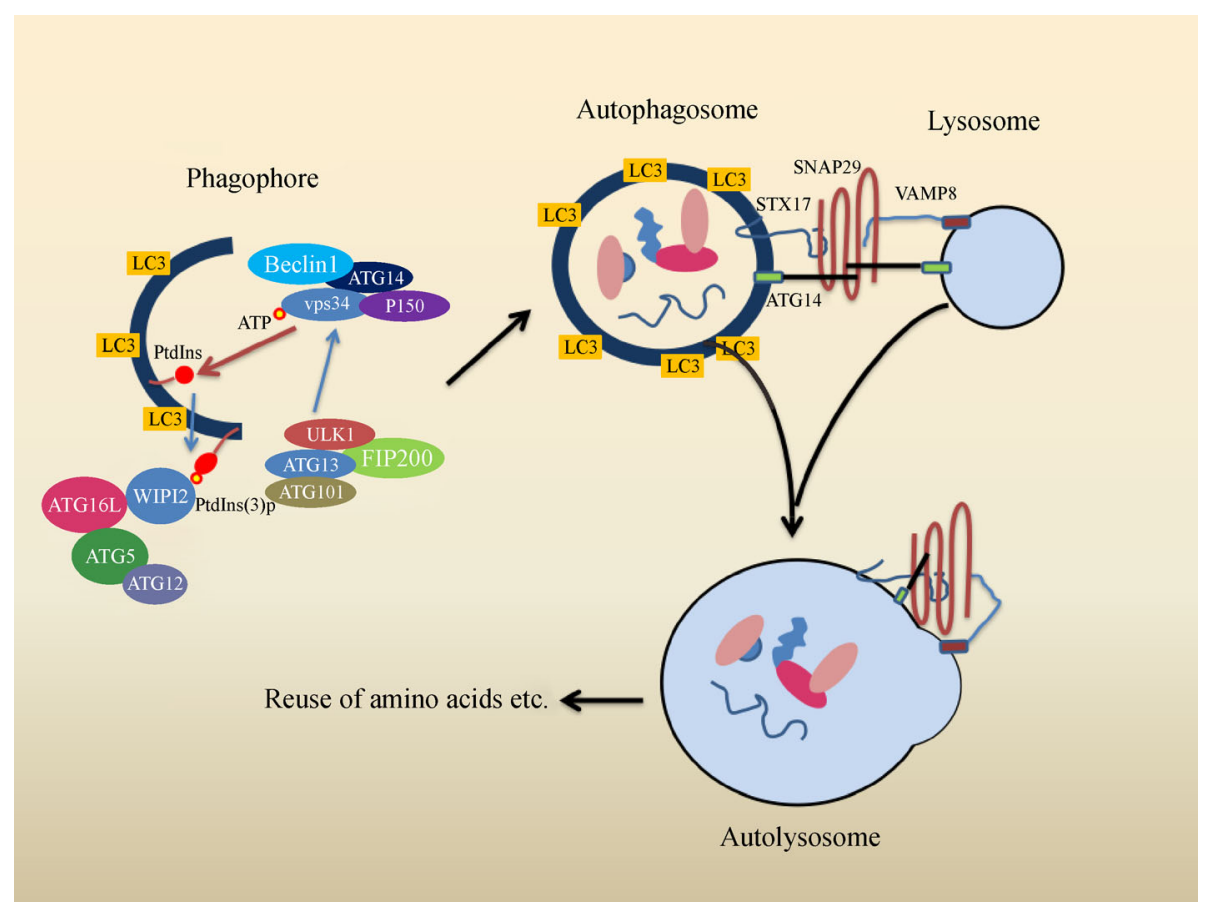

Fig. 1 Molecular mechanism of autophagosome initiation and maturation 


\subsection{Autophagosome formation}

Upon induction of autophagy, the immediate event is the formation of the phagophore to supply the platform for ATGs. Yet, the original membrane source of phagophore remains unclear. Some findings have shown that the autophagosome contains lipids of Golgi and plasma membranes ${ }^{[17,18]}$. Many molecules have been founded to be involved in autophagosome formation. The preautophagosomal structure (PAS) has been described in yeast ${ }^{[19]}$. The PAS is a single punctuate structure close to the yeast vacuole membrane, where almost all ATG proteins are colocalized. Same structures are also found in mammalian cells ${ }^{[20]}$. The recruitment of ATG proteins to the phagophore along with the acquisition of lipids expands the membrane to form a cup-shaped precursor of the autophagosome termed the omegasome ${ }^{[21]}$.

During autophagosome formation, many genes are assembled into core autophagy machinery. This core machinery is composed of three major functional groups: (1) ATG9 and its cycling system, which includes ATG9, the ATG1/ULK1 kinase complex, ATG2 and ATG18; (2) the VPS34 complex; and (3) the ATG8/LC3 and ATG12 ubiquitin-like protein system, ATG7, ATG10 and ATG3, ATG4, ATG5 and ATG16 ${ }^{[1]}$. ATG9 is the only transmembrane protein in the core machinery in different species ${ }^{[22]}$; it cycles from multiple peripheral sites, which hints at the role of carrying membrane for phagophore expansion. ATG9 is capable of self-interaction and may exist in a complex ${ }^{[23]}$ and the population of ATG9 molecules is localized on the PAS ${ }^{[19]}$. In addition, ATG1 complex, ATG2, and ATG18 are required for retrieval of ATG9 from PAS. The VPS34 complex presumably functions at the PAS by recruiting phosphatidylinositol(3)-phosphate (PI3P) binding proteins such as ATG18 ${ }^{[24,25]}$ which participate in ATG9 retrieval. During autophagy, a population of ATG8-PE (phosphatidylethanolamine), needs to be released from PE by ATG4 ${ }^{[26]}$. ATG12-modified ATG5 forms a multimeric complex with ATG16 ${ }^{[27]}$, which decorates the expanding phagophore with ATG8-PE ${ }^{[28,29]}$. Upon the formation of PAS and recruitment of proteins, the delivery process of the proteins to the PAS is mediated by an adaptor, ATG11. After completion of autophagosome formation, both the cargo and the receptor are delivered to the vacuole, where the receptor is degraded (Fig. 1) ${ }^{[30]}$.

\subsection{Autophagosome maturation}

The autophagic double membrane engulfs its cargo and closes. This is then followed by fusion with the lysosome and delivery of the content for digestion. Recent studies have implicated SNARE proteins (soluble N-ethylmaleimide-sensitive factor attachment protein receptor) including vesicle-associated membrane proteins (VAMP7,
VAMP3 and VAMP8), Qa-SNARE syntaxin 17 (STX17), SNAP-29 and vesicle transport interacting t-SNAREs homolog 1B (Vtilb). These proteins mediate the fusion of autophagosome and lysosome ${ }^{[31-34]}$. STX17 is localized on the outer membrane of closed autophagosomes and interacts with SNAP-29 and VAMP8. Depletion of STX17 blocks the autophagosomes and lysosome fusion which leads to accumulation of autophagosome in the cytosol ${ }^{[34]}$. In addition, ATG14 (also known as BECN1-associated autophagy-related key regulator, Barkor or ATG14L), binds to the STX17-SNAP29 binary t-SNARE complex on autophagosomes and promotes VAMP8 interaction ${ }^{[35]}$. The COPs (CoCrMo metal particles) ${ }^{[36]}$ and ESCRT III (endosomal complex required for transport III) complex are necessary for proper autophagosomal degradation ${ }^{[37]}$. ESCRT III complex is a core complex in multivesicular body sorting. The HOPS complex (homotypic fusion and protein sorting $)^{[38]}$ is also involved in the fusion process. During autophagosome fusion with lysosome, LAMP-2 (lysosomal associated membrane protein 2), which decorates the lysosome membrane, seems to be involve in the process. LAMP-2 knockout mice have been shown to have an impaired phagosomal maturation in neutrophils LAMP proteins ${ }^{[39]}$. Rab7 (Ras-related protein 7) as one of the small GTPase Rab proteins acts at a critical point of the maturation process of both autophagosomes and endosomes. Inhibition of Rab7 disrupts lysosome biogenesis, accumulation of autophagic vacuoles, and finally inhibition of autophagic vacuole fusion ${ }^{[40]}$. Rab7 GTPase activity is elevated by the VPS34 complex with BECN1 and UVRAG which promotes autophagosome fusion with late endosomes/lysosomes ${ }^{[41]}$. However, another complex that includes BECN1, RUNICON, VPS34, hVps15, and UVRAG may function in the maturation of autophagosomes. It has been seen that the knockdown of Rubicon results in a dramatic increase of the autophagosomes ${ }^{[42]}$. TECPR1 (tectonin beta-propeller repeat containing 1) is an ATG5-interacting protein and a component of the autophagy network and plays a critical role in autophagosome maturation. TECPR1 may function in promoting fusion of autophagosomes with lysosomes through its association of ATG12-ATG5 and PI3P, which will initiate autophagosome-lysosome fusion (Fig. 1) ${ }^{[43]}$.

\section{The core complexes in nutrient stress-induced autophagy}

Autophagy is a lysosomal-dependent cellular degradation process. The initiation of autophagosome requires activity of ULK1 protein kinase and the VPS34 lipid kinase to recruit the ATGs to the isolation membrane for the autophagosome to elongate and curve. The regulatory mechanism and pathway of these two complexes are critical for the autophagy induction process. Cellular stress 
such as starvation signals the initiation of the induction process. Amino acid and energy withdrawal are associated with mTOR and AMPK activity, which is significant in autophagy.

\subsection{Metabolic master regulator: mTOR and AMPK}

\subsection{1 mTOR}

Cells can sense different environments and respond by proliferation, differentiation or just quiescence. In most conditions, the content of growth factors, amino acids or energy are the critical signals that affect the cells' physiology.

The highly conserved Ser/Thr protein kinase target of rapamycin (TOR) is a most important sensor in amino acid signaling. TOR can control the cell metabolic level and proliferation. Mammalian TOR (mTOR) forms two different complexes in mammals: mTOR complex 1 (mTORC1) and complex 2 (mTORC2). But only mTORC1 can sense the dynamic of both growth factors and amino acids. When the environmental growth factors and the amino acids exist, the mTORC1 will activate and transfer anabolism signals to the downstream effector to promote cell protein synthesis and cell growth ${ }^{[44]}$. The concentration of certain amino acids, such as leucine, arginine, glutamine, have an affect mTORC1 activity directly ${ }^{[45]}$. The mTORC1 consist of mTOR, regulatoryassociated protein of mTOR (raptor), which functions in substrate recognition and recruitment, mammalian lethal with Sec13 protein 8 (mLST8), proline-rich AKT substrate $40 \mathrm{kDa}$ (PRAS40) and DEP-domain-containing mTORinteracting protein (Deptor). This components have different functions in regulating mTORC1 activity. PRAS40 and Deptor can inhibit the mTORC1 activity when bound to the complex. When the cellular amino acid or growth factors concentrations increase, amino acids can stimulate mTOR to phosphorylate both PRAS40 and Deptor, which will release PRAS40 and Deptor from the complexes $^{[46]}$.

In the process of controlling protein synthesis, mTORC1 is the most powerful. The eukaryotic translation initiation factor 4E (eIF4E) binding protein 1 (4E-BP1) and S6 kinase 1 (S6K1) are two other important proteins regulating protein synthesis. The phosphorylation of 4EBP1 directly by mTORC1 sets eIF4E free to participate in the formation of the eIF4F complex that is required for the initiation of cap-dependent translation. The activation of S6K1 by mTORC1 can increase mRNA biogenesis, as well as translational initiation and elongation. mTORC1 can also activate the regulatory element tripartite motifcontaining protein-24 (TIF-1A) to interact with RNA Polymerase I (Pol I) and the synthesis of rRNA (rRNA) to increase the protein synthesis ${ }^{[47]}$.

\subsubsection{AMPK}

Numerous studies have found that AMPK acts as a central energy sensor to sense and regulate cellular activities in response to many cellular stresses such as energy withdrawal. AMPK exists as heterotrimeric complexes consisting of a catalytic $\alpha$ subunit and two regulatory subunits $\beta$ and $\gamma$. Environmental AMP, ADP, or ATP can directly bind to AMPK via the adenine nucleotide binding sites of the $\gamma$ subunit and binding of AMP or ADP activates AMPK $^{[48]}$.

The canonical mechanisms of activation of mammalian AMPK, involve increases in the ratio of AMP/ATP and ADP/ATP, by starvation of glucose or oxygen, as well as by stresses which lead to ATP consumption. Activation of AMPK can promotes glucose uptake into cells. Moreover, AMPK promotes uptake into cardiac myocytes via translocation of vesicles containing the fatty acid transporter CD36 (cluster of differentiation 36) to the plasma membrane ${ }^{[49]}$. Another crucial process activated by AMPK is mitochondrial biogenesis ${ }^{[50]}$.

Other studies have demonstrated new mechanism of AMPK control of the expression of genes involved in energy metabolism by coordination with another metabolic sensor, the $\mathrm{NAD}^{+}$-dependent type III deacetylase SIRT1 (silent mating type information regulation 2 homolog 1). AMPK enhances SIRT1 activity by increasing cellular $\mathrm{NAD}^{+}$levels which leads to deacetylation and modulation of the activity of downstream SIRT1 targets that include the peroxisome proliferator-activated receptor- $\gamma$ coactivator $1 \alpha(\mathrm{PGC}-1 \alpha)$ and the forkhead box O1 (FOXO1) and O3 (FOXO3a) transcription factors. The AMPK-induced SIRT1-mediated deacetylation of these targets explains many of the convergent biological effects of AMPK and SIRT1 on energy metabolism ${ }^{[51]}$.

3.2 Nutrient signals regulate autophagy activity through coordination with the autophagy core complexes

\subsubsection{ATG1/ULK1 complexes}

Atg1 is a serine-threonine kinase in yeast and is important in the induction of autophagy. The Unc-51-like kinase 1 (ULK1) is the homolog of yeast ATG1 and appears to be closely related. The siRNA knockdown of ULK1 blocks autophagy in HEK293 cells $^{[52]}$. ULK1 deletion, or expression of a kinase-dead mutant, leads to the accumulation of most other components of the autophagy machinery at the PAS as well as abrogation of autophagy initiation ${ }^{[53]}$. The ULK complex is recruited to the membrane of the autophagosome during the early stage of autophagy induction. This downstream reactivation, or sustained activation of the ULK complex at later stages, supports a self-reinforcing cycle between the ULK complex and the 
class III PI3-kinase complex driving expansion of the autophagosome initiated at the omegasome ${ }^{[54]}$. ATG1 forms a complex with ATG13 and ATG17. The phosphorylation of ATG13 can directly affect its binding to ATG1 and ATG1 $7^{[55]}$. The ULK1 complexes have been identified recently and consist of mammalian ATG13 ${ }^{[56]}$, FIP200 [focal adhesion kinase (FAK) family interacting protein of $200 \mathrm{kD}]^{[57]}$ and ATG101 ${ }^{[58]}$. FIP200 is considered to have functional similarities to yeast ATG17 $7^{[59]}$, although, FIP200 and ATG101 in mammals are not found in the yeast, and homologs of ATG17 in yeast have not been found in mammals. Differences between the components of the ATG1 complex in yeast versus mammals indicate that the mechanism of autophagy initiation may differ from one species to another ${ }^{[60]}$. ATG13 mediates the interaction of ULK1 and FIP200 ${ }^{[61]}$ and there is evidence that ULK1 can also directly bind FIP200. Whereas, ATG13 and FIP200 can elevate ULK1 kinase activity ${ }^{[52]}$.

\subsubsection{ULK1 complexes respond to nutrients status}

Multiple studies have shown the inhibitory function of the mTOR complex 1 (mTORC1) in autophagy ${ }^{[62]}$. The highly conserved serine-threonine protein kinase TOR is a master kinase of the amino acid pool sensing signaling and mTORC1 promotes cell growth by accelerating the biosynthetic process and inhibiting catabolic processes ${ }^{[63]}$. In yeast, upon autophagy initiation, the activity of the ULK1-ATG13-ATG17 complex can be mediated by TOR for the initiation of autophagosome formation ${ }^{[64]}$. In mammalian cells, ATG13 and ULK1 can be directly phosphorylated by mTOR to inhibit autophagy initiation. Autophosphorylation of ULK1 increased under mTOR inhibitory conditions, and ULK-dependent phosphorylation of FIP200 was induced by mTOR inhibition, which implies that mTOR functions as a negative factor ${ }^{[52]}$. In addition, serine 757 in ULK1 has been identified as the phosphorylated site for mTORC1 $1^{[65]}$. The mTOR can also inhibit autophagy through phosphorylating AMBRA1 (autophagy/BECN-1 regulator 1), which is a ULK1 binding partner required for ULK1 stability and kinase activity. Upon amino acid withdrawal, AMBRA1 is dephosphorylated. This dephosphorylated AMBRA1 then interacts with the E3-ligase TRAF6, promotes ULK1 ubiquitylation and its subsequent stabilization, self-association and function. Furthermore, activated mTORC1 can indirectly inhibit ULK1 stability and activity by modifying AMBRA1 and impairing its E3-ligaserelated functions ${ }^{[66]}$.

AMPK senses the ratio of AMP/ATP and increases ATP levels through accelerated catabolism. Cellular AMP, ADP or ATP can directly bind to the AMPK $\gamma$ subunit ${ }^{[67]}$. Under energy deprivation, AMPK is activated to promote autophagy activity ${ }^{[68]}$ by increased ULK1 autophosphorylation. Two phosphorylation sites, serine 317 and 777, in
ULK1 have been identified when glucose withdrawal causes activation of AMPK ${ }^{[65]}$. Overexpression of Rheb, an mTORC1 activator, can decrease ULK1-AMPK binding. Consistently, the binding of ULK1 and AMPK depends on inhibition of mTORC $1^{[65]}$ A feedback mechanism involving ULK1 is also observed which inhibits AMPK through phosphorylation ${ }^{[69]}$. This ULK1 feedback mechanism toward AMPK controls the autophagy to sustain a suitable level even in the inductive stage and this ULK1 feedback mechanism regulates the level of autophagy.

\subsubsection{VPS34 complexes}

VPS34 is a class III PI3K that phosphorylates the D-3 position of phosphatidylinositol to produce PI3P. Phosphatidylinositol is recruited to the cell membrane once phosphorylated to PI3P. It then recruits PI3P binding proteins to the membrane to modulate membrane activity ${ }^{[70]}$. Yeast genetic and biochemical studies have shown that VPS34 can form different complexes ${ }^{[71,72]}$. In yeast, complex I consists of VPS34, VPS15, ATG6/VPS30 and ATG14. The ATG6/VPS30 unit interacts with VPS38 to form the distinctive VPS34 complex II which is required for endosome-to-Golgi retrograde trafficking ${ }^{[73]}$. In mammalian cells, the primary components of theVPS34 complexes include VPS34 and p150 (VPS15), BECN1 and ATG14/Barkor for complex I or UVRAG (UV radiation resistance associated) for complex $\mathrm{II}^{[74]}$. Other components of VPS34 have been also identified: VMP1 (vacuole membrane protein 1 , interacts with BECN1, a mammalian autophagy initiator, through the VMP1-ATG domain, which is essential for autophagosome formation ${ }^{[75]}$ ), AMBRA1 (activating molecule in BECN1-regulated autophagy, a positive regulator of autophagy ${ }^{[76]}$, Bif-1 (endophilin B1, positive mediator of VPS3 $4^{[77]}$ ) and Rubicon (RUN and cysteine rich domain containing BECN1 interacting protein, which reduces VPS34 activity and downregulates autophagy ${ }^{[78]}$ ).

\subsubsection{VPS34 complexes regulated by nutrient signaling}

The VPS34 complex phosphorylates PI to form PI3P, which allows PI3P binding proteins on the membrane to modulate membrane activity. The regulatory activity of VPS34 is critical for autophagosome initiation. The function of mTOR has been well established as the cell growth executor as well as being amino acid and growth factor sensor and mTOR can inhibit the autophagic degradative pathway by inhibition of ULK1 complexe function as well as VPS34 activity. Recently, studies showed mTORC1 inhibits the activity of the ATG14containing VPS34 complex by phosphorating ATG14, which is required for PIK3C3 inhibition by mTORC1 both in vitro and in vivo ${ }^{[79]}$. Amino acid starvation or treatment 
with the mTORC1 inhibitor rapamycin and Torin1 results in an increase of activity of VPS34 complex. Consistently, cotransfection of RHEB (Ras homolog enriched in brain), an activator of mTORC1, dramatically reduces the activity of the ATG14-containing PIK3C3 complex. However, further treatment with rapamycin or Torin1 reversed this effect $^{[79]}$. The mTORC1 mediates the amino acid signal to regulate ATG14-associated PIK3C3 activity and autophagy. Methionine deficiency results in reduced protein synthesis, alters the mTOR/S6K1 signaling pathway ${ }^{[80]}$. Moreover, in a case of methionine-choline deficiency, which induce nonalcoholic steatohepatitis in mice, will trigger autophagy ${ }^{[81]}$. Further experiments identified five serine and threonine sites (serine 3, 223, 383 and 440, and threonine 233) that are phosphorylated by mTORC1 in vitro ${ }^{[79]}$. SLC38A9, an uncharacterized protein with sequence similarity to amino acid transporters, is a lysosomal transmembrane protein and loss of SLC38A9 represses $\mathrm{mTORC} 1$ activation by amino acids, particularly arginine. Overexpression of SLC38A9 or just its Ragulator binding domain makes mTORC1 signaling insensitive to amino acid starvation ${ }^{[82]}$. In the VPS34 complex II, mTOR can phosphorylate UVRAG at serine 550 and 571. Serine to alanine substitutions at serine 550 and 571 prevent the phosphorylation of UVRAG by mTOR. Thus, mTORmediated UVRAG phosphorylation at serine 550 and 571 enhances VPS34 activity, and the phosphorylation of UVRAG is essential for the concomitant PI3P production and increased PIK3C 3 activity ${ }^{[83]}$.

AMPK, the energy status sensor, has a key role in regulating different VPS34 complexes. Glucose starvation results in the depletion of energy and activation of AMPK as well as the induction of autophagy. During glucose starvation, VPS34 complex activity increases in AMPK $\alpha 1 / 2$ double-knockout cells. However, VPS34 complex activity is decreased in AMPK wild type cells. When VPS34 complexes that are free of ATG14 and UVRAG are phosphorylated at threonine 163 and serine 165 by AMPK their activity is inhibited ${ }^{[44]}$. Notably, serine 91 and 94 of BECN1, rather than VPS34 phosphorylation, are required for activation of VPS34 complex by AMPK containing ATG14. Consistently, the complex containing a phosphorylation-mimetic double substitution mutant of BECN1 at serine 91 and 94 showed higher basal activity even in glucose-rich conditions. ATG14 enhances the phosphorylation activation of BECN1, and suppresses the inhibitory phosphorylation of VPS34 by AMPK. Ectopic expression of ATG14 strongly promoted BECN1 phosphorylation and inhibited VPS34 phosphorylation and AMPK co-expres$\operatorname{sion}^{[84]}$.

\section{Conclusions}

Autophagy is a catabolic pathway degrading proteins and organelles to recycle nutrients which is induced when cells face stress or starvation. Upon induction of autophagy, two complexes: ULK1 and VPS34 are recruited to autophagic membranes to initiate the membrane extension and are closely regulated by two master metabolic switches: mTORC1 and AMPK. mTORC1 and AMPK are the key regulatory factors in protein and amino acid or energy metabolism respectively. mTORC1 is activated under conditions of amino acid or growth factors stimulation, on the other hand, it is suppressed by amino acid starvation and autophagy is induced. AMPK, a crucial cellular energy sensor, is activated when energy deprivation occurs, which triggers autophagy. More studies are needed to determine the functional connections between autophagy and metabolic pathways, to elucidate the mechanisms of these metabolic physiological processes and disease responses.

Acknowledgements The financial support from the China Scholarship Council, the National Basic Research Program of China (2013CB117301), the National Natural Science Foundation of China (31272448, 31472101, 31420103908, and 31528018), the 111 Project (B16044), Beijing Nova Program (xx2013055), Education Foundation of China Agricultural University "Dabeinong Education Fund" (1041-2415001), and National Department Public Benefit Research Foundation (201403047) are gratefully acknowledged

Compliance with ethics guidelines Long $\mathrm{He}$, Shabnam Eslamfam, Xi Ma, and Defa Li declare that they have no conflicts of interest or financial conflicts to disclose.

This article is a review and does not contain any studies with human or animal subjects performed by any of the authors.

\section{References}

1. Xie Z, Klionsky D J. Autophagosome formation: core machinery and adaptations. Nature Cell Biology, 2007, 9(10): 1102-1109

2. Mizushima N, Levine B, Cuervo A M, Klionsky D J. Autophagy fights disease through cellular self-digestion. Nature, 2008, 451 (7182): 1069-1075

3. Renna M, Bento C F, Ghislat G, Puri C, Ashkenazi A, Vicinanza M, Menzies F M, Rubinsztein D C. Mammalian autophagy-how does it work? Annual Review of Biochemistry, 2016 (first published online)

4. Kamada Y, Funakoshi T, Shintani T, Nagano K, Ohsumi M, Ohsumi Y. Tor-mediated induction of autophagy via an Apg1 protein kinase complex. Journal of Cell Biology, 2000, 150(6): $1507-1513$

5. Kim J, Kim Y C, Fang C, Russell R C, Kim J H, Fan W, Liu R, Zhong Q, Guan K L. Differential regulation of distinct Vps34 complexes by AMPK in nutrient stress and autophagy. Cell, 2013, 152(1-2): 290-303

6. Kuma A, Hatano M, Matsui M, Yamamoto A, Nakaya H, Yoshimori T, Ohsumi Y, Tokuhisa T, Mizushima N. The role of autophagy during the early neonatal starvation period. Nature, 2004, 432 (7020): 1032-1036

7. Zhang S, Li X, Li L, Yan X. Autophagy up-regulation by early weaning in the liver, spleen and skeletal muscle of piglets. British Journal of Nutrition, 2011, 106(2): 213-217 
8. Kuma A, Hatano M, Matsui M, Yamamoto A, Nakaya H, Yoshimori T, Ohsumi Y, Tokuhisa T, Mizushima N. The role of autophagy during the early neonatal starvation period. Nature, 2004, 432 (7020): 1032-1036

9. Komatsu M, Waguri S, Ueno T, Iwata J, Murata S, Tanida I, Ezaki J, Mizushima N, Ohsumi Y, Uchiyama Y, Kominami E, Tanaka K, Chiba T. Impairment of starvation-induced and constitutive autophagy in Atg7-deficient mice. Journal of Cell Biology, 2005, 169(3): 425-434

10. Rabinowitz J D, White E. Autophagy and metabolism. Science, 2010, 330(6009): 1344-1348

11. Deretic V, Levine B. Autophagy, immunity, and microbial adaptations. Cell Host \& Microbe, 2009, 5(6): 527-549

12. Levine B, Mizushima N, Virgin H W. Autophagy in immunity and inflammation. Nature, 2011, 469(7330): 323-335

13. Jia K, Thomas C, Akbar M, Sun Q, Adams-Huet B, Gilpin C, Levine B. Autophagy genes protect against Salmonella typhimurium infection and mediate insulin signaling-regulated pathogen resistance. Proceedings of the National Academy of Sciences of the United States of America, 2009, 106(34): 14564-14569

14. Kuballa P, Huett A, Rioux J D, Daly M J, Xavier R J. Impaired autophagy of an intracellular pathogen induced by a Crohn's disease associated ATG16L1 variant. PLoS ONE, 2008, 3(10): e3391

15. Wild P, Farhan H, McEwan D G, Wagner S, Rogov V V, Brady N R, Richter B, Korac J, Waidmann O, Choudhary C, Dötsch V, Bumann D, Dikic I. Phosphorylation of the autophagy receptor optineurin restricts Salmonella growth. Science, 2011, 333(6039): 228-233

16. Benjamin J L, Sumpter R Jr, Levine B, Hooper L V. Intestinal epithelial autophagy is essential for host defense against invasive bacteria. Cell Host \& Microbe, 2013, 13(6): 723-734

17. Ravikumar B, Moreau K, Jahreiss L, Puri C, Rubinsztein D C. Plasma membrane contributes to the formation of pre-autophagosomal structures. Nature Cell Biology, 2010, 12(8): 747-757

18. Yamamoto A, Masaki R, Tashiro Y. Characterization of the isolation membranes and the limiting membranes of autophagosomes in rat hepatocytes by lectin cytochemistry. Journal of Histochemistry and Cytochemistry, 1990, 38(4): 573-580

19. Suzuki K, Kirisako T, Kamada Y, Mizushima N, Noda T, Ohsumi Y. The pre-autophagosomal structure organized by concerted functions of APG genes is essential for autophagosome formation. EMBO Journal, 2001, 20(21): 5971-5981

20. Itakura E, Mizushima N. Characterization of autophagosome formation site by a hierarchical analysis of mammalian Atg proteins. Autophagy, 2010, 6(6): 764-776

21. Simonsen A, Stenmark H. Self-eating from an ER-associated cup. Journal of Cell Biology, 2008, 182(4): 621-622

22. Noda T, Kim J, Huang W, Baba M, Tokunaga C, Ohsumi Y, Klionsky D J. Apg9p/Cvt7p is an integral membrane protein required for transport vesicle formation in the Cvt and autophagy pathways. Journal of Cell Biology, 2000, 148(3): 465-480

23. Reggiori F, Shintani T, Chong H, Nair U, Klionsky D J. Atg9 cycles between mitochondria and the pre-autophagosomal structure in yeasts. Autophagy, 2005, 1(2): 101-109

24. Guan J, Stromhaug P E, George M D, Habibzadegah-Tari P, Bevan A, Dunn W A Jr, Klionsky D J. Cvt18/Gsa12 is required for cytoplasm-to-vacuole transport, pexophagy, and autophagy in
Saccharomyces cerevisiae and Pichia pastoris. Molecular Biology of the Cell, 2001, 12(12): 3821-3838

25. Matsuura A, Tsukada M, Wada Y, Ohsumi Y. Apg1p, a novel protein kinase required for the autophagic process in Saccharomyces cerevisiae. Gene, 1997, 192(2): 245-250

26. Kirisako T, Ichimura Y, Okada H, Kabeya Y, Mizushima N, Yoshimori T, Ohsumi M, Takao T, Noda T, Ohsumi Y. The reversible modification regulates the membrane-binding state of Apg8/Aut7 essential for autophagy and the cytoplasm to vacuole targeting pathway. Journal of Cell Biology, 2000, 151(2): 263-276

27. Mizushima N, Kuma A, Kobayashi Y, Yamamoto A, Matsubae M, Takao T, Natsume T, Ohsumi Y, Yoshimori T. Mouse Apg16L, a novel WD-repeat protein, targets to the autophagic isolation membrane with the Apg12-Apg5 conjugate. Journal of Cell Science, 2003, 116(9): 1679-1688

28. Kabeya Y, Mizushima N, Ueno T, Yamamoto A, Kirisako T, Noda T, Kominami E, Ohsumi Y, Yoshimori T. LC3, a mammalian homologue of yeast Apg8p, is localized in autophagosome membranes after processing. EMBO Journal, 2000, 19(21): 57205728

29. Mizushima N, Yamamoto A, Hatano M, Kobayashi Y, Kabeya Y, Suzuki K, Tokuhisa T, Ohsumi Y, Yoshimori T. Dissection of autophagosome formation using Apg5-deficient mouse embryonic stem cells. Journal of Cell Biology, 2001, 152(4): 657-668

30. Kim J, Kamada Y, Stromhaug P E, Guan J, Hefner-Gravink A, Baba M, Scott S V, Ohsumi Y, Dunn W A Jr, Klionsky D J. Cvt9/Gsa9 functions in sequestering selective cytosolic cargo destined for the vacuole. Journal of Cell Biology, 2001, 153(2): 381-396

31. Takáts S, Nagy P, Varga Á, Pircs K, Kárpáti M, Varga K, Kovács A L, Hegedűs K, Juhász G. Autophagosomal Syntaxin17-dependent lysosomal degradation maintains neuronal function in Drosophila. Journal of Cell Biology, 2013, 201(4): 531-539

32. Atlashkin V, Kreykenbohm V, Eskelinen E L, Wenzel D, Fayyazi A, Fischer von Mollard G. Deletion of the SNARE vtilb in mice results in the loss of a single SNARE partner, syntaxin 8. Molecular and Cellular Biology, 2003, 23(15): 5198-5207

33. Fader C M, Sánchez D G, Mestre M B, Colombo M I. TI-VAMP/ VAMP7 and VAMP3/cellubrevin: two v-SNARE proteins involved in specific steps of the autophagy/multivesicular body pathways. Biochimica et Biophysica Acta, 2009, 1793(12): 1901-1916

34. Itakura E, Kishi-Itakura C, Mizushima N. The hairpin-type tailanchored SNARE syntaxin 17 targets to autophagosomes for fusion with endosomes/lysosomes. Cell, 2012, 151(6): 1256-1269

35. Diao J, Liu R, Rong Y, Zhao M, Zhang J, Lai Y, Zhou Q, Wilz L M, Li J, Vivona S, Pfuetzner R A, Brunger A T, Zhong Q. ATG14 promotes membrane tethering and fusion of autophagosomes to endolysosomes. Nature, 2015, 520(7548): 563-566

36. Razi M, Chan E Y, Tooze S A. Early endosomes and endosomal coatomer are required for autophagy. Journal of Cell Biology, 2009, 185(2): 305-321

37. Lee J A, Beigneux A, Ahmad S T, Young S G, Gao F B. ESCRT-III dysfunction causes autophagosome accumulation and neurodegeneration. Current Biology, 2007, 17(18): 1561-1567

38. Nickerson D P, Brett C L, Merz A J. Vps-C complexes: gatekeepers of endolysosomal traffic. Current Opinion in Cell Biology, 2009, 21 (4): 543-551 
39. Eskelinen E L, Illert A L, Tanaka Y, Schwarzmann G, Blanz J, von Figura K, Saftig P. Role of LAMP-2 in lysosome biogenesis and autophagy. Molecular Biology of the Cell, 2002, 13(9): 33553368

40. Hyttinen J M T, Niittykoski M, Salminen A, Kaarniranta K. Maturation of autophagosomes and endosomes: a key role for Rab7. Biochimica et Biophysica Acta, 2013, 1833(3): 503-510

41. Liang C, Lee J S, Inn K S, Gack M U, Li Q, Roberts E A, Vergne I, Deretic V, Feng P, Akawa C, Jung J U. Beclin1-binding UVRAG targets the class $\mathrm{C}$ Vps complex to coordinate autophagosome maturation and endocytic trafficking. Nature Cell Biology, 2008, 10 (7): 776-787

42. Matsunaga K, Saitoh T, Tabata K, Omori H, Satoh T, Kurotori N, Maejima I, Shirahama-Noda K, Ichimura T, Isobe T, Akira S, Noda $\mathrm{T}$, Yoshimori T. Two Beclin 1-binding proteins, Atg14L and Rubicon, reciprocally regulate autophagy at different stages. Nature Cell Biology, 2009, 11(4): 385-396

43. Chen D, Fan W, Lu Y, Ding X, Chen S, Zhong Q. A mammalian autophagosome maturation mechanism mediated by TECPR1 and the Atg12-Atg5 conjugate. Molecular Cell, 2012, 45(5): 629-641

44. Jewell J L, Russell R C, Guan K L. Amino acid signalling upstream of mTOR. Nature Reviews Molecular Cell Biology, 2013, 14(3): 133-139

45. Avruch J, Long X, Ortiz-Vega S, Rapley J, Papageorgiou A, Dai N. Amino acid regulation of TOR complex 1. American Journal of Physiology: Endocrinology and Metabolism, 2009, 296(4): 592602

46. Oshiro N, Takahashi R, Yoshino K I, Tanimura K, Nakashima A, Eguchi S, Miyamoto T, Hara K, Takehana K, Avruch J, Kikkawa U, Yonezawa K. The proline-rich Akt substrate of $40 \mathrm{kDa}$ (PRAS40) is a physiological substrate of mammalian target of rapamycin complex 1. Journal of Biological Chemistry, 2007, 282(28): 20329-20339

47. Laplante M, Sabatini D M. mTOR signaling in growth control and disease. Cell, 2012, 149(2): 274-293

48. Hardie D G. AMP-activated protein kinase - an energy sensor that regulates all aspects of cell function. Genes \& Development, 2011, 25(18): 1895-1908

49. Habets D D, Coumans W A, El Hasnaoui M, Zarrinpashneh E, Bertrand L, Viollet B, Kiens B, Jensen T E, Richter E A, Bonen A, Glatz J F. Crucial role for LKB1 to AMPKa2 axis in the regulation of CD36-mediated long-chain fatty acid uptake into cardiomyocytes. Biochimica et Biophysica Acta, 2009, 1791(3): 212-219

50. Winder W W, Holmes B F, Rubink D S, Jensen E B, Chen M, Holloszy J O. Activation of AMP-activated protein kinase increases mitochondrial enzymes in skeletal muscle. Journal of Applied Physiology, 2000, 88(6): 2219-2226

51. Cantó C, Gerhart-Hines Z, Feige J N, Lagouge M, Noriega L, Milne J C, Elliott P J, Puigserver P, Auwerx J. AMPK regulates energy expenditure by modulating $\mathrm{NAD}^{+}$metabolism and SIRT1 activity. Nature, 2009, 458(7241): 1056-1060

52. Ganley I G, Lam D H, Wang J, Ding X, Chen S, Jiang X. ULK1ATG13-FIP200 complex mediates mTOR signaling and is essential for autophagy. Journal of Biological Chemistry, 2009, 284(18): 12297-12305

53. Papinski D, Kraft C. Regulation of autophagy by signaling through the Atg1/ULK1 complex. Journal of Molecular Biology, 2016, 428 (9): 1725-1741

54. Ktistakis N T, Tooze S A. Digesting the expanding mechanisms of autophagy. Trends in Cell Biology, 2016, (first published online)

55. Reggiori F, Tucker K A, Stromhaug P E, Klionsky D J. The Atg1Atg13 complex regulates Atg9 and Atg23 retrieval transport from the pre-autophagosomal structure. Developmental Cell, 2004, 6(1): 79-90

56. Kamada Y, Funakoshi T, Shintani T, Nagano K, Ohsumi M, Ohsumi Y. Tor-mediated induction of autophagy via an Apg1 protein kinase complex. Journal of Cell Biology, 2000, 150(6): $1507-1513$

57. Chan E Y, Longatti A, McKnight N C, Tooze S A. Kinaseinactivated ULK proteins inhibit autophagy via their conserved Cterminal domains using an Atg13-independent mechanism. Molecular and Cellular Biology, 2009, 29(1): 157-171

58. Noda N N, Mizushima N. Atg101: not just an accessory subunit in the autophagy-initiation complex. Cell Structure and Function, 2016, 41(1): 13-20

59. Mercer C A, Kaliappan A, Dennis P B. A novel, human Atg13 binding protein, Atg101, interacts with ULK1 and is essential for macroautophagy. Autophagy, 2009, 5(5): 649-662

60. Hara T, Takamura A, Kishi C, Iemura S I, Natsume T, Guan J L, Mizushima N. FIP200, a ULK-interacting protein, is required for autophagosome formation in mammalian cells. Journal of Cell Biology, 2008, 181(3): 497-510

61. Mizushima N. The role of the Atg1/ULK1 complex in autophagy regulation. Current Opinion in Cell Biology, 2010, 22(2): 132-139

62. Hosokawa N, Hara T, Kaizuka T, Kishi C, Takamura A, Miura Y, Iemura S I, Natsume T, Takehana K, Yamada N, Guan J L, Oshiro $\mathrm{N}$, Mizushima N. Nutrient-dependent mTORC1 association with the ULK1-Atg13-FIP200 complex required for autophagy. Molecular Biology of the Cell, 2009, 20(7): 1981-1991

63. Jung C H, Ro S H, Cao J, Otto N M, Kim D H. mTOR regulation of autophagy. FEBS Letters, 2010, 584(7): 1287-1295

64. Shintani T, Klionsky D J. Autophagy in health and disease: a double-edged sword. Science, 2004, 306(5698): 990-995

65. Kim J, Kundu M, Viollet B, Guan K L. AMPK and mTOR regulate autophagy through direct phosphorylation of Ulk1. Nature Cell Biology, 2011, 13(2): 132-141

66. Hardie D G. AMP-activated protein kinase-an energy sensor that regulates all aspects of cell function. Genes \& Development, 2011, 25(18): 1895-1908

67. Nazio F, Strappazzon F, Antonioli M, Bielli P, Cianfanelli V, Bordi M, Gretzmeier C, Dengjel J, Piacentini M, Fimia G M, Cecconi F. mTOR inhibits autophagy by controlling ULK1 ubiquitylation, selfassociation and function through AMBRA1 and TRAF6. Nature Cell Biology, 2013, 15(4): 406-416

68. Liang J, Shao S H, Xu Z X, Hennessy B, Ding Z, Larrea M, Kondo S, Dumont D J, Gutterman J U, Walker C L, Slingerland J M, Mills G B. The energy sensing LKB1-AMPK pathway regulates p27kip1 phosphorylation mediating the decision to enter autophagy or apoptosis. Nature Cell Biology, 2007, 9(2): 218-224

69. Löffler A S, Alers S, Dieterle A M, Keppeler H, Franz-Wachtel M, Kundu M, Campbell D G, Wesselborg S, Alessi D R, Stork B. Ulk1mediated phosphorylation of AMPK constitutes a negative 
regulatory feedback loop. Autophagy, 2011, 7(7): 696-706

70. Obara K, Ohsumi Y. PtdIns 3-kinase orchestrates autophagosome formation in yeast. Journal of Lipids, 2011, 2011: 498768

71. Kametaka S, Okano T, Ohsumi M, Ohsumi Y. Apg14p and Apg6/ $\mathrm{Vps} 30 \mathrm{p}$ form a protein complex essential for autophagy in the yeast, Saccharomyces cerevisiae. Journal of Biological Chemistry, 1998, 273(35): 22284-22291

72. Obara K, Sekito T, Ohsumi Y. Assortment of phosphatidylinositol 3-kinase complexes-Atg14p directs association of complex I to the pre-autophagosomal structure in Saccharomyces cerevisiae. Molecular Biology of the Cell, 2006, 17(4): 1527-1539

73. Chen J, Li Y, Tian Y, Huang C, Li D, Zhong Q, Ma X. Interaction between microbes and host intestinal health: modulation by dietary nutrients and gut-brain-endocrine-immune axis. Current Protein \& Peptide Science, 2015, 16(7): 572-603

74. Fan P, Li L, Rezaei A, Eslamfam S, Che D, Ma X. Metabolites of dietary protein and peptides by intestinal microbes and their impacts on gut. Current Protein \& Peptide Science, 2015, 16(7): 646-654

75. Ropolo A, Grasso D, Pardo R, Sacchetti M L, Archange C, Re A L, Seux M, Nowak J, Gonzalez C D, Iovanna J L, Vaccaro M I. The pancreatitis-induced vacuole membrane protein 1 triggers autophagy in mammalian cells. Journal of Biological Chemistry, 2007, 282(51): 37124-37133

76. Fimia G M, Stoykova A, Romagnoli A, Giunta L, Di Bartolomeo S, Nardacci R, Corazzari M, Fuoco C, Ucar A, Schwartz P, Gruss P. Ambra1 regulates autophagy and development of the nervous system. Nature, 2007, 447(7148): 1121-1125

77. Takahashi Y, Coppola D, Matsushita N, Cualing H D, Sun M, Sato Y, Liang C, Jung J U, Cheng J Q, Mul J J, Pledger W J, Wang H G. Bif-1 interacts with Beclin 1 through UVRAG and regulates autophagy and tumorigenesis. Nature Cell Biology, 2007, 9(10): 1142-1151

78. Zhong Y, Wang Q, Li X, Yan Y, Backer J M, Chait B T, Heintz N, Yue Z. Distinct regulation of autophagic activity by $\operatorname{Atg} 14 \mathrm{~L}$ and Rubicon associated with Beclin 1-phosphatidylinositol-3-kinase complex. Nature Cell Biology, 2009, 11(4): 468-476

79. Yuan H, Russell R C, Guan K L. Regulation of PIK3C3/VPS34 complexes by MTOR in nutrient stress-induced autophagy. Autophagy, 2013, 9(12): 1983-1995

80. Tang Y, Tan B, Xiong X, Li F, Ren W, Kong X, Qiu W, Hardwidge $\mathrm{P}$ R, Yin Y. Methionine deficiency reduces autophagy and accelerates death in intestinal epithelial cells infected with enterotoxigenic Escherichia coli. Amino Acids, 2015, 47(10): 2199-2204

81. Chen R, Wang Q, Song S, Liu F, He B, Gao X. Protective role of autophagy in methionine-choline deficient diet-induced advanced nonalcoholic steatohepatitis in mice. European Journal of Pharmacology, 2016, 5(770): 126-133

82. Wang S, Tsun Z Y, Wolfson R L, Shen K, Wyant G A, Plovanich M E, Yuan E D, Jones T D, Chantranupong L, Comb W, Wang T, BarPeled L, Zoncu R, Straub C, Kim C, Park J, Sabatini B L, Sabatini D M. Lysosomal amino acid transporter SLC38A9 signals arginine sufficiency to mTORC1. Science, 2015, 347(6218): 188-194

83. Munson M J, Allen G F, Toth R, Campbell D G, Lucocq J M, Ganley I G. mTOR activates the VPS34-UVRAG complex to regulate autolysosomal tubulation and cell survival. EMBO Journal, 2015, 34(17): 2272-2290

84. Ma X, Chen J, Tian Y. Pregnane X receptor as the "sensor and effector" in regulating epigenome. Journal of Cellular Physiology, 2015, 230(4): 752-757 